Money and the Decentralization of Exchange

Author(s): Joseph M. Ostroy and Ross M. Starr

Source: Econometrica, Vol. 42, No. 6 (Nov., 1974), pp. 1093-1113

Published by: The Econometric Society

Stable URL: http://www.jstor.org/stable/1914220

Accessed: 19/07/2011 15:33

Your use of the JSTOR archive indicates your acceptance of JSTOR's Terms and Conditions of Use, available at http://www.jstor.org/page/info/about/policies/terms.jsp. JSTOR's Terms and Conditions of Use provides, in part, that unless you have obtained prior permission, you may not download an entire issue of a journal or multiple copies of articles, and you may use content in the JSTOR archive only for your personal, non-commercial use.

Please contact the publisher regarding any further use of this work. Publisher contact information may be obtained at http://www.jstor.org/action/showPublisher?publisherCode=econosoc.

Each copy of any part of a JSTOR transmission must contain the same copyright notice that appears on the screen or printed page of such transmission.

JSTOR is a not-for-profit service that helps scholars, researchers, and students discover, use, and build upon a wide range of content in a trusted digital archive. We use information technology and tools to increase productivity and facilitate new forms of scholarship. For more information about JSTOR, please contact support@jstor.org. 


\title{
MONEY AND THE DECENTRALIZATION OF EXCHANGE
}

\author{
By Joseph M. Ostroy and Ross M. StarR ${ }^{1}$
}

\begin{abstract}
A pairwise trading process is formulated subject to conditions of non-negativity of traders' holdings, quid pro quo, and a limited number of trading opportunities. The following points are made : (i) there is a centralized procedure that achieves the equilibrium allocation for an arbitrary economy; (ii) it is not in general possible to find a decentralized procedure that achieves the equilibrium allocation for an arbitrary economy; and (iii) in a monetary economy there is a decentralized procedure that achieves the equilibrium allocation.

The usefulness of money is that it allows decentralization of the trading process.
\end{abstract}

\section{THE PROCESS OF TRADE}

AN EMINENT SCHOLAR has related to us an anecdote elaborating the "inconveniences of barter:"

Consider the eminent scholar travelling far from home. He stops at a hotel and asks for lodging for the night. The clerk replies, "That will be fifteen dollars (unit of account)." E.S. agrees and extracts from the trunk of his car a copy of his latest textbook. "Here's a copy of my latest textbook. It sells for fifteen dollars (unit of account)." "Good, here's your room key. Have a pleasant stay." The hotel keeper trades the book for fifteen dollars' worth of soap. The soap distributor sends the book as payment for detergent, to a detergent manufacturer. The latter pays the book, as dividend, to a stockholder. The stockholder sends the book, as allowance to his son, studying at a major university where E.S.'s text is used in a large lecture course. The boy trades the book to a student in the course in exchange for fifteen dollars' worth of contraband, which he consumes.

That is how trade would take place in an ideally coordinated barter economy. The need for such coordination arises from the restriction that goods when received must be paid for by a corresponding opposite delivery of goods of equal value, quid pro quo. The origins of this restriction are strategic. Without a quid pro quo constraint, agents would not be effectively prevented from violating their budget constraints.

The inconveniences of barter consist in the information and coordination implicit in the story at each stage of trade. Only if the hotel keeper knows that his distributor's supplier will accept textbooks in trade is he likely to accept E.S.'s book in exchange for lodging. To make a substantial number of transactions depend on trading partners' demands, trading partners' trading partners' demands, trading partners' trading partners'... trading partners' demands, would make even the simplest trade depend on the communication of massive

\footnotetext{
${ }^{1}$ The research described in this paper was supported by grants from the Ford Foundation, the National Science Foundation, and the University of California Board of Regents. Collaboration was initiated while Professor Starr was consultant to the Western Management Science Institute, UCLA, whose support and that of its director, Jacob Marschak, is gratefully acknowledged. We are deeply indebted to Gordon Bradley for providing the essential step in the proof of Lemma 1. We have also benefited from discussions with Robert Clower, Peter Howitt, Herbert Scarf, and James Tobin. Errors are the authors' responsibility.
} 
amounts of data about who trades with whom, when, and what they want. As long as there is a generally acceptable, universally held medium of exchange, no such communication is necessary. Each trade merely consists in the exchange of a desired commodity for the medium of exchange. All one need know about one's trading partners' trading partners is that, like everyone else, they accept the medium of exchange. The informational requirements of barter imply the need for a central coordination of trade; the function of a common medium of exchange is to allow decentralization of the trading process.

\section{A MODEL OF THE TRADING PROCESS}

Let there be $N$ commodities indexed by $n=1, \ldots, N$ and a set of $J$ traders, $I$, indexed by $i=1, \ldots, J$. Let each trader have an endowment bundle $b_{i} \in R_{+}^{N} .^{2}$ Trader $i$ 's net trade vector is $z_{i}$. The complex of initial endowments will be represented by the $J \times N$ matrix $B=\left\|b_{i n}\right\|$. Similarly, let $Z=\left\|z_{i n}\right\|$.

For the purposes of this paper, an exchange economy will be characterized by a price vector $p=\left(p_{1}, \ldots, p_{N}\right)$, a matrix of excess demands, and a matrix of initial endowments, $(p, Z, B)$, which satisfy the following, and only the following restrictions :

$$
\begin{array}{ll}
Z p=0, & \text { where } p_{n}>0 \\
\sum z_{i}=0, & \text { and } \\
Z \geqslant-B & \text { (the inequality holds entry-wise). }
\end{array}
$$$$
(n=1, \ldots, N),
$$

Condition (U.1) says that at the (strictly positive) prices $p$, the value of a trader's supplies and demands are equal. Conditions (U.2) and (U.3) guarantee that aggregate trades and individual trades, respectively, are feasible.

A competitive equilibrium of an exchange economy is the most interesting case of $(p, Z, B)$ fulfilling (U). A competitive equilibrium must satisfy (U) and, for all $i, z_{i}$ must be maximal for his preferences among all allowable excess demands. However, because the problem addressed here is the implementation, not the existence, of a competitive equilibrium and because any $(p, Z, B)$ is a competitive equilibrium for some pattern of preferences, the qualification that $z_{i}$ be maximal can be ignored. ${ }^{3}$

No theory of price formation is advanced here. It is assumed that the process of price formation occurs prior to the process of exchange to be discussed below. A supporting hypothesis is as follows: the Walrasian auctioneer announces equilibrium prices but leaves the expedition of trades to the individuals themselves. This common knowledge of prices will be used to define quid pro quo in

\footnotetext{
${ }^{2} R_{+}^{N}$ is the non-negative orthant of $N$-dimensional Euclidean space.

${ }^{3}$ If $p$ is an equilibrium price vector, there may be many distinct $Z$ 's such that $z_{i}$ is an element of $i$ 's excess demand correspondence and $(p, Z, B)$ fulfills (U). Since the focus of this study is the implementation of an allocation, $Z$, it is necessary first to specify which of a multiplicity of possible allocations is to be implemented. Hence the use of a specific $Z$ rather than a correspondence. This represents, of course, an indeterminancy that recurs throughout general equilibrium theory.
} 
exchange, a standard of behavior traders will impose on each other. Elsewhere, it has been shown that if any one trader does not enforce quid pro quo and accepts an exchange which decreases the value of his endowment at these prices while the other traders refused to make such trades, his final trading position will suffer. ${ }^{4}$

Trade is supposed to take place between one trader and another, in pairs. If we adopt the convention that a trader can be a member of only one pair at a time, it requires $\tau$ periods for each trader to form a pair with every other trader, once and only once, where

$$
\tau=\left\{\begin{array}{l}
J-1 \quad \text { if } J \text { is even, } \\
J \quad \text { if } J \text { is odd }{ }^{5}
\end{array}\right.
$$

In the analysis below, we take the order in which pairs meet to be arbitrary. The order of meetings is described by $\left\{\pi^{t}\right\}, t=1, \ldots, \tau$, a sequence of permutations of the set $I$, so that (i) $\pi^{t}(i)=j$ if and only if $\pi^{t}(j)=i$, all $i \in I$ and $t=1, \ldots, \tau$; (ii) $\pi^{t}(i) \neq i$, all $i$ and $t$ if $J$ is even, and $\pi^{t}(i)=i$ for exactly one $t$, each $i \in I$, if $J$ is odd; and (iii) $\pi^{t}(i) \neq \pi^{s}(i)$, all $i \in I$ and $s \neq t$. These three conditions guarantee that for all $i, j \in I$, there is precisely one $t, 1 \leqslant t \leqslant \tau$, such that $\pi^{t}(i)=j$. Call such a sequence, $\left\{\pi^{t}\right\}$, a round. Any such sequence will do in that none of our results depend on more than (i), (ii), and (iii).

At the start of the $t$ th period, trader $i$ 's holdings will be represented by $w_{i}^{t}$ with $w_{i}^{1}=b_{i}$. The change in $i$ 's holdings between $t$ and $t+1, a_{i}^{t}=w_{i}^{t+1}-w_{i}^{t}$, is the trade $i$ performs in period $t$. The matrix of trades in $t$ is $A^{t}=\left\|a_{i n}^{t}\right\|=\left\|w_{i n}^{t+1}\right\|-$ $\left\|w_{i n}^{t}\right\|=W^{t+1}-W^{t}$. Trader $i$ 's hitherto unsatisfied excess demands on entering period $t$ are $v_{i}^{t}=v_{i}^{1}-\Sigma_{T=1}^{T=t-1} a_{i}^{T}$, with $v_{i}^{1}=z_{i}$.

Let $\pi^{t}(i)=j$. Consider the meeting and trade between $i$ and $j$. Each brings his holdings, $w_{i}^{t}$ and $w_{j}^{t}$, to the pair. Positive entries in the vector $a_{i}^{t}$ indicate goods going from $j$ to $i$ and negative entries, goods going from $i$ to $j$. After trading, $i$ 's holdings will be $w_{i}^{t+1}=w_{i}^{t}+a_{i}^{t}$ and $j$ 's will be $w_{j}^{t+1}=w_{j}^{t}+a_{j}^{t}$. We place the following three restrictions on $a_{i}^{t}$ and $a_{j}^{t}$ :

$$
w_{i}^{t}+a_{i}^{t} \geqslant 0, \quad w_{j}^{t}+a_{j}^{t} \geqslant 0 \quad \text { (non-negativity of holdings). }
$$

$$
a_{i}^{t}=-a_{j}^{t} \quad \text { (conservation of commodities). }
$$

Should trades fulfill (A) for all $i, j \in I$ and $t=1, \ldots, \tau$, we shall say that the sequence of trades is admissible.

The non-negativity requirement, (A.1), says that a trader can at no time have a negative holding of any commodity. A trader cannot deliver to his partner more of a commodity than he currently holds. This may be interpreted as a prohibition on the issue of I.O.U.'s. ${ }^{6}$

\footnotetext{
${ }^{4}$ See $[9$, Section IV].

${ }^{5}$ See $[9]$ for some properties of a model of bilateral exchange where each trader meets with only a fraction of the other traders.

${ }^{6}$ In a model including futures markets, (A.1) does not prevent a trader from selling futures contracts for goods which do not yet exist, but to which he has title.
} 
The conservation condition, (A.2), says that in the process of trade, commodities are neither created nor destroyed. Goods delivered are received and vice versa.

The quid pro quo condition, (A.3), requires that in the trade between $i$ and $j$ each delivers to the other goods of equal value. Full payment is made for value received where goods are evaluated at equilibrium prices.

Conditions (A.1) and (A.2) are feasibility restrictions defining bilateral exchange. The origins of (A.3) are behavioral; if $A^{t}$ were proposed such that $p \cdot a_{i}^{t}<0$ for some $i$, then $i$ would refuse to trade.

Given prices, an order of meetings for the pairs of traders, and an admissible sequence of trades, the outcome can be described as the resulting allocation of goods among traders. At the end of one round the outcome is $W^{\tau+1}=B+$ $\Sigma_{t=1}^{\tau} A^{t}$. We will say that full execution of excess demands $Z$ has been achieved in one round if

$$
\sum_{t=1}^{t=\tau} A^{t}=Z
$$

Should time run out $(t=\tau)$ before all demands are fulfilled and supplies delivered, (E) will not be satisfied.

To illustrate, let the order of meetings in a four-person economy be $(\overline{12}, \overline{34})$, $(\overline{14}, \overline{23})$, and $(\overline{13}, \overline{24})$ in periods 1,2 , and 3 , respectively, where $\overline{i j}$ indicates that $i$ and $j$ form a pair. Then if $p=(1,1,1,1)$ and initial excess demands and endowments are given by the matrices $Z$ and $B$, where rows denote traders and columns denote commodities,

$$
Z=\left[\begin{array}{rrrr}
1 & -1 & 0 & 0 \\
0 & 1 & -1 & 0 \\
0 & 0 & 1 & -1 \\
-1 & 0 & 0 & 1
\end{array}\right], \quad B=\left[\begin{array}{llll}
0 & 1 & 0 & 0 \\
0 & 0 & 1 & 0 \\
0 & 0 & 0 & 1 \\
1 & 0 & 0 & 0
\end{array}\right]
$$

trades fulfilling $(\mathrm{A})$ and $(\mathrm{E})$ may be found. If the last period is dropped, either (A) or (E) may be satisfied, but not both.

Will (E) be fulfilled without violating (A)? To answer this we must specify what information traders have at each trading opportunity.

A trading rule is a function that tells each pair what trade to make. The inputs for the decision are not only what they have on hand - which defines what they can do-but what they know. Indeed, what they have on hand is just a part of what they know. There is a substantial variety of alternative assumptions and out of these we choose to examine extreme cases. Define a trading rule as a function

$$
\rho\left(w_{i}^{t}, w_{j}^{t} \mid L^{t}\right)=\left(a_{i}^{t}, a_{j}^{t}\right)
$$

where $\pi^{t}(i)=j$ and $L^{t}$ is the set of information, beyond their current holdings, available to the pair at date $t$. 
A rule is thought of as decentralized if it does not require more information than $i, j$ are likely to possess. More precisely, varying degrees of modest informational requirements are characterized as follows:

(D.1) $\quad L^{t}=\left\{\left(v_{i}^{t}, v_{j}^{t}\right)\right\}$,

(D.2) $\quad L^{t}=\left\{\left(v_{i}^{t}, v_{j}^{t}\right),(i, j)\right\}$,

(D.3) $\quad L^{t}=\left\{\left(v_{i}^{t}, v_{i}^{t-1}, \ldots, v_{i}^{1} ; v_{j}^{t}, v_{j}^{t-1}, \ldots, v_{j}^{1}\right),(i . j)\right\}$.

(D.1) says that the decision on what $i$ and $j$ trade depends on $i$ and $j$ 's currently unsatisfied excess demands and supplies. Equation (D.2) says that the decision may depend not only on current excess demands and supplies, but on who $i$ and $j$ are, their names, as well. Equation (D.3) says that in addition the decision may depend on $i$ 's and $j$ 's trading histories.

The notion of decentralization advanced here is a suitable extension of that concept to the problem under consideration. An economic arrangement is generally described as decentralized if it involves individual agents making decisions based on a fairly small body of universally communicated information (e.g., prices) and on information which the agents themselves may be supposed to possess (e.g., individual tastes and endowments and, in this case, the pair's trading history). (D) applies these restrictions to trading rules for pairs of agents. ${ }^{7}$

A trading rule is centralized if it requires more information than (D.1), (D.2), or (D.3) can provide. It will suffice to represent the informational requirements of a centralized rule by

$$
L^{t}=\left\{\left(v_{1}^{t}, v_{1}^{t-1}, \ldots, v_{1}^{1} ; \quad v_{2}^{t}, \ldots, ; \quad v_{J}^{t}, v_{J}^{t-1}, \ldots, v_{J}^{1}\right), \quad(i, j)\right\} .
$$

If $\rho$ requires $(\mathrm{C})$, then $i$ and $j$ 's trading decision depends on the trading histories of everyone in the economy, not only those of $i$ and $j$.

The role of money as a medium of exchange consists in allowing full execution to be achieved in one round by a decentralized rule, whereas, in the absence of money, full execution requires more time, or a centralized rule, or sufficient quantities of non-money commodities. ${ }^{8}$ These contentions are embodied in the following results.

THEOREM 1: There is a trading rule which, for all $(p, Z, B)$ satisfying $(U)$, satisfies $(A),(C)$, and $(E)$.

${ }^{7}$ According to (D.3), traders know or remember only part of the trading histories they may previously have known and used to make trading decisions. A rule making full use of trading history data (precluded by (D.3)) would allow traders to make their trading decisions not only on the basis of their previous trades, their current partner's previous trades, but as well on their previous partners' previous trades, ..., etc. With this information each trader would be able to make more precise estimates of the probable excess demands of future partners and this would certainly allow the traders to come closer to full execution. But the record-keeping and complexity that such a rule would require appears to be so great as to be unfeasible, or at least very costly.

${ }^{8}$ The role of inventories of non-money commodities was brought to our attention by R. W. Clower, who pointed out Theorem 3 below. 
THEOREM 2: There is no trading rule which, for all $(p, Z, B)$ satisfying $(U)$, satisfies $(A),(D .3)$, and $(E)$.

THEOREM 3: Let $(p, Z, B)$ satisfy $(U)$; further let there be a trader $j \in I$ with $b_{j} \geqslant \Sigma_{i \neq j}\left[z_{i}\right]^{+} .9$ Then there is a trading rule which satisfies $(A),(D .2)$, and $(E)$.

THEOREM 4: Let $(p, Z, B)$ satisfy $(U)$; further let there be a commodity $m, 1 \leqslant m \leqslant$ $N$, so that $p_{m} b_{i m} \geqslant p \cdot\left[z_{i}\right]^{+}-p_{m}\left[z_{i m}\right]^{+}$for all $i \in I$. Then there is a trading rule which satisfies $(A),(D .1)$, and $(E)$.

Theorems 1 and 2 demonstrate the trade-off between full execution and the limited information. Together, they say that although there exists a rule which makes (A) and (E) compatible for every competitive equilibrium $(p, Z, B)$, that rule must be centralized. Theorems 3 and 4 demonstrate the trade-off between full execution and the presence of inventories. They say that if there is enough slack in initial endowments - either a trader whose endowments are sufficient to fulfill all others' excess demands (Theorem 3), or a commodity such that the value of each trader's holdings of it is at least equal to the value of his planned purchases of all other commodities (Theorem 4) - decentralized trading is compatible with full execution. In particular, the commodity $m$ in Theorem 4 is regarded as money, and it behaves as money in the trading rule used to prove that theorem.

These results are conditional on bilateral trading opportunities being limited to precisely one round. Extending the model to include additional rounds, e.g., as many as there are traders, it can be shown that neither inventories nor information beyond that contained in (D), are necessary to make (A) and (E) compatible. Thus, there is a trade-off between time and information or inventories in achieving full execution.

\section{IDEAL COORDINATION IN A BARTER ECONOMY}

This section is devoted to a proof of Theorem 1. The strategy for the proof is as follows: (i) define a chain as an elementary configuration of excess demands, (ii) show that there is a centralized rule which achieves full execution of a chain in one round, (iii) show that any equilibrium configuration of excess demands may be represented as the sum of a finite number of chains, and (iv) apply the rule developed in (ii) to the chains in (iii) and show that the trade consisting of the sum of the trades prescribed for each of the chains is admissible and achieves full execution.

Let $\left\{i_{1}, i_{2}, \ldots, i_{s}\right\}$ be a subset of the traders called the members of the chain, $\left\{n_{1}, n_{2}, \ldots, n_{s}\right\}$ a subset of the commodities, and let $\delta$ be a positive scalar. Denote by $\left[\left(i_{1} n_{1} i_{2} n_{2} \ldots i_{s} n_{s} i_{1}\right), p, \delta\right]$ that configuration of excess demands where (i) $i_{1}$ has excess supply of $n_{1}$ for which $i_{2}$ has excess demand, $i_{2}$ has excess supply of $n_{2}$

\footnotetext{
${ }^{9}$ For a vector $x=\left(x_{1}, \ldots, x_{N}\right),[x]^{-} \equiv\left(\min \left(x_{1}, 0\right), \ldots, \min \left(x_{N}, 0\right)\right)$ and $[x]^{+} \equiv\left(\max \left(x_{1}, 0\right), \ldots\right.$, $\left.\max \left(x_{N}, 0\right)\right)$.
} 
for which $i_{3} \ldots$, and $i_{s}$ has excess supply of $n_{s}$ for which $i_{1}$ has excess demand; and (ii) the value of the commodity each individual demands is equal at the prices, $p$, to the value of the commodity he supplies, and is equal to $\delta$.

Call $\left[\left(i_{1} n_{1} i_{2} \ldots i_{s} n_{s} i_{1}\right), p, \delta\right]$ a chain of length $s$ and width $\delta$. By definition $2 \leqslant s \leqslant$ $\min (J, N)$ when $J$ is the number of traders and $N$ is the number of commodities.

A matrix representation of the chain $\left[\left(i_{1} n_{1} i_{2} \ldots i_{s} n_{s} i_{1}\right), p, \delta\right]$ will be denoted by the $J \times N$ matrix $\tilde{Z}=\left\|\tilde{\Sigma}_{i n}\right\|$ where

$$
\tilde{z}_{i n}=\left\{\begin{array}{ccr}
-\delta / p_{n} & \text { if }(i, n)=\left(i_{r}, n_{r}\right) & (r=1, \ldots, s), \\
\delta / p_{n} & \text { if }(i, n)=\left(i_{r}, n_{r-1}\right) & \left(r=2, \ldots, s \text { or }(i, n)=\left(i_{1}, n_{s}\right)\right), \\
0 & \text { otherwise. }
\end{array}\right.
$$

A positive entry indicates demand and a negative entry supply of a commodity. An example of a matrix representation of a chain of length 4 and width 1 is given by the numerical illustration of $Z$ in Section 2, above. There, $i_{r}=r, r=1, \ldots, 4$ and $n_{r}=r+1, r=1,2,3$, and $n_{4}=1$.

There is an equivalence between chains and cyclic permutations. By relabelling the indices on traders and commodities in the chain $\left[\left(i_{1} n_{1} i_{2} \ldots i_{s} n_{s} i_{1}\right), p, \delta\right]$ we may obtain $i_{h}=n_{h}=h, h=1, \ldots, s$. Then, ignoring $p$ and $\delta$, the interdependencies among the members of a chain of length $s$ may be represented by the cyclic permutation

$$
\alpha=\left(\begin{array}{llll}
1 & 2 & \ldots & s \\
2 & 3 & \ldots & 1
\end{array}\right) \cdot{ }^{10}
$$

The first line lists the "names" of the traders in the chain, and the second line contains the same list in a different order. The appearance of " 2 " under " 1 " denotes that trader 1 gives commodity 1 to trader $2, \ldots$, and " 1 " under " $s$ " that trader $s$ gives commodity $s$ to trader 1 .

Suppose trader 1 and $r, 1<r \leqslant s$, meet and exchange their excess supplies. What change in the pattern of interdependence occurs as a result of this trade? The exchange is a transposition of the excess supplies of traders 1 and $r$ and is represented by the permutation

$$
\beta=\left(\begin{array}{llllll}
1 & 2 & \ldots & r & \ldots & s \\
r & 2 & \ldots & 1 & \ldots & s
\end{array}\right) .
$$

The effect of this trade on the original chain is given by premultiplying $\alpha$ by $\beta$ :

$$
\beta \cdot \alpha=\left(\begin{array}{cccccccc}
1 & 2 & \ldots & r-1 & r & r+1 & \ldots & s \\
2 & 3 & \ldots & 1 & r+1 & r+2 & \ldots & r
\end{array}\right) .
$$

To verify, for example, the last column, note that $\alpha(s)=1$ and $\beta(1)=r$, so $\beta \cdot \alpha(s)=r$. Notice that $\beta \cdot \alpha$ consists of two disjoint chains (disjoint cyclic permutations).

\footnotetext{
${ }^{10}$ The definition of a cyclic permutation and the above double-rowed method of representing it is contained in most introductory texts on algebra. A standard reference is [7].
} 
One is of the first $r-1$ traders, and the other is of the last $s-(r-1)$ traders. This result provides us with an elementary proof of the following lemma:

LEMMA 1: Let $(p, \tilde{Z}, B)$ satisfy $(U)$ and suppose $\tilde{Z}$ is the matrix representation of the chain $\left[\left(i_{1} n_{1} i_{2} \ldots i_{s} n_{s} i_{1}\right), p, \delta\right]$. Then there is a trading rule satisfying $(A)$, $(C)$, and $(E)$.

ProOF: It suffices to prove the existence of a rule satisfying (A) and (E) since such a rule, if it exists, can require no more than complete information which is (C).

The proof is by induction on the length of the chain, now denoted by $k$. For $k=2$, (A) and (E) follow if the pair exchange excess supplies when they meet.

Suppose there is a rule satisfying (A) and (E) for all $k, k \leqslant s-1<\min (J, N)$. From the demonstration of the relation between chains and cyclic permutations we may denote a typical chain of length $s$ by $\alpha$, above.

Let $\bar{t}$ be the first period in which any pair of traders from $\{1, \ldots, s\}$ meet. Without loss of generality, assume $\pi^{i}(1)=r, 1<r \leqslant s$. Let this pair exchange their excess supplies. The result is to transform the original chain into two smaller disjoint chains of lengths $(r-1)$ and $(s-(r-1))$. Since $\bar{t}$ is the first period in which any of the set $\{1, \ldots, s\}$ meet and since $\left\{\pi^{t}\right\}$ permits all pairs to meet, there is a $t$, $\bar{t} \leqslant t \leqslant \tau$ such that every pair within each disjoint chain meet. Apply the induction hypothesis to each of these chains, whose lengths are less than or equal to $s-1$, to obtain the desired conclusion. ${ }^{11}$

Q.E.D.

Not every non-zero matrix of excess demands in $(U)$ is a chain. However, every such matrix can be written as a sum of chains. We shall show that any exchange economy represented by $(p, Z, B)$ satisfying $(\mathrm{U})$ can be broken down into components $\left(p, \widetilde{Z}^{k}, B^{k}\right)$ each of which satisfy $(\mathrm{U})$ and $\Sigma \widetilde{Z}^{k}=Z, \Sigma B^{k}=B$.

As a simple illustration, suppose $p=(1,1,1)$ and

$$
Z=\left(\begin{array}{rrr}
8 & -7 & -1 \\
-3 & 9 & -6 \\
-5 & -2 & 7
\end{array}\right), \quad B=\left(\begin{array}{rrr}
0 & -7 & -1 \\
-3 & 0 & -6 \\
-5 & -2 & 0
\end{array}\right)
$$

Note that $Z$ is not itself a chain. However,

$$
\begin{array}{ll}
\tilde{Z}^{1}=\left(\begin{array}{rrr}
1 & 0 & -1 \\
0 & 0 & 0 \\
-1 & 0 & 1
\end{array}\right), & \tilde{Z}^{2}=\left(\begin{array}{rrr}
4 & -4 & 0 \\
0 & 4 & -4 \\
-4 & 0 & 4
\end{array}\right), \\
\tilde{Z}^{3}=\left(\begin{array}{rrr}
0 & 0 & 0 \\
0 & 2 & -2 \\
0 & -2 & 2
\end{array}\right), & \tilde{Z}^{4}=\left(\begin{array}{rrr}
3 & -3 & 0 \\
-3 & 3 & 0 \\
0 & 0 & 0
\end{array}\right),
\end{array}
$$

${ }^{11}$ Lemma 1 is simply a slight refinement of an elementary result in the theory of groups that every cyclic permutation (called here a chain) can be represented as a product of transpositions (called here bilateral trades). See [7, p. 94]. The refinement amounts to showing that any permutation can be represented as the product of a fixed list of cycles, where each cycle is used at most once. 
are matrix representations of chains and $\tilde{Z}^{1}+\tilde{Z}^{2}+\tilde{Z}^{3}+\tilde{Z}^{4}=Z$. If we set $B^{k}=-\left[\tilde{Z}^{k}\right]^{-}$, then $\Sigma B^{k}=B$ and $\left(p, \tilde{Z}^{k}, B^{k}\right), k=1,2,3,4$ is the desired decomposition.

The general proposition is as follows:

Lemma 2: Let $(p, Z, B)$ satisfy $(U)$ and $Z \neq 0$. Then there exist $\left(p, \widetilde{Z}^{k}, B^{k}\right), k=$ $1, \ldots, K$ satisfying $(U)$ and, (i) $\widetilde{Z}^{k}$ is the matrix representation of a chain at prices $p$, all $k$; (ii) $Z=\widetilde{Z}^{k}, B=\Sigma B^{k}$.

Proof: Since $Z \neq 0$ satisfies (U.2), there are traders, $i_{1}$ and $i_{2}$, and a commodity, $n_{1}$, such that $z_{i_{1} n_{1}}<0$ and $z_{i_{2} n_{1}}>0$. Since (U.1) holds, there must be a commodity $n_{2}$ such that $z_{i_{2} n_{2}}<0$. Continue in this manner to order traders and commodities such that $z_{i_{s} n_{s}}<0$ and $z_{i_{s} n_{s-1}}>0$ as long as $i_{s} \neq i_{r}, r=1, \ldots, s-1$ and/or $n_{s} \neq n_{r-1}, r=2, \ldots, s-1$. However, (U.1) and (U.2) insure that there is some $s \leqslant \min (J, N)$ with $i_{s}=i_{r}$ or $n_{s}=n_{r-1}$.

(i) If $i_{s}=i_{r}$, then we have

$$
\left(p^{n_{r}}\right)\left(z_{i_{r} n_{r}}\right)<0, \quad\left(p^{n_{r}}\right)\left(z_{i_{r+1} n_{r}}\right)>0, \quad \ldots, \quad\left(p^{n_{s-1}}\right)\left(z_{i_{s-1} n_{s-1}}\right)<0,
$$

and

$$
\left(p^{n_{s}-1}\right)\left(z_{i_{r} n_{s-1}}\right)>0 .
$$

Let $\delta$ be the minimum of the absolute values of these amounts. Then we may form the chain $\left[\left(i_{r} n_{r} i_{r+1} \ldots i_{s-1} n_{s-1} i_{r}\right), p, \delta\right]$.

(ii) If $n_{s}=n_{r-1}$, then we have

$$
\left(p^{n_{r}}\right)\left(z_{i_{r} n_{r}}\right)<0, \quad\left(p^{n_{r}}\right)\left(z_{i_{r+1} n_{r}}\right)>0, \quad \ldots, \quad\left(p^{n_{r-1}}\right)\left(z_{i_{s} n_{r-1}}\right)<0,
$$

and

$$
\left(p^{n_{r-1}}\right)\left(z_{i_{r} n_{r-1}}\right)>0 .
$$

Let $\delta$ be the minimum of the absolute values of these amounts. Then we may form the chain $\left[\left(i_{r} n_{r} i_{r+1} \ldots i_{s} n_{r-1} i_{s}\right), p, \delta\right]$.

In either case let $\widetilde{Z}^{1}$ be the matrix representation of the resulting chain. By construction, $\operatorname{sign} \tilde{z}_{i n}^{1}=\operatorname{sign} z_{i n}$. Further, $Z-\tilde{Z}^{1}$ satisfies (U.1) and (U.2) and has at least one fewer non-zero entry than $Z$. To find $\widetilde{Z}^{2}$ apply to $Z-\widetilde{Z}^{1}$ the procedure used to derive $\tilde{Z}^{1}$. To find $\tilde{Z}^{l}$, apply the procedure to $Z-\sum_{k=1}^{k=l-1} \tilde{Z}^{k}$. This will guarantee that $\operatorname{sign} z_{i n}^{l}=\operatorname{sign} z_{i n}$ and that $Z-\Sigma_{k=1}^{k=l} \tilde{Z}^{k}$ has at least one fewer non-zero entry than $Z-\sum_{k=1}^{k=l-1} \widetilde{Z}^{k}$. Since $Z$ has at most $J N$ non-zero entries, the procedure must terminate-i.e., $Z-\sum_{k=1}^{k=K} \widetilde{Z}^{k}=0$-for some $K \leqslant J N$.

It only remains for us to find $B^{k}$ such that $\left(p, \widetilde{Z}^{k}, B^{k}\right)$ satisfy (U.3) and $\Sigma B^{k}=B$. This may be done by setting $B^{k}=-\left[\tilde{Z}^{k}\right]^{-}$, which implies $\widetilde{Z}^{k} \geqslant-B^{k}, k=1, \ldots$, $K-1$, and by setting $B^{K}=B-\sum_{k=1}^{k=K-1}\left(-\left[\tilde{Z}^{k}\right]^{-}\right)=B+Z-\tilde{Z}^{K}$. Since $B+Z$ $\geqslant 0, \tilde{Z}^{K} \geqslant-B^{K}$, and therefore, $\Sigma_{k=1}^{k=K} B^{k}=B$.

Q.E.D.

Lemma 2 permits the extension of the trading rule shown to exist in Lemma 1 to any equilibrium matrix of excess demands which may now be viewed as a sum of 
chains. It has already been shown that for any chain $\tilde{Z}^{k}$ there is a sequence of admissible trades, denoted by $\left\{A^{k t}\right\}, t=1, \ldots, \tau$ guaranteeing full execution.

THEOREM 1: Let $(p, Z, B)$ satisfy $(U)$. There is a trading rule satisfying $(A),(C)$, and $(E)$.

Proof: Let $\left\{A^{k t}\right\}$ be the trades prescribed by the trading rule shown to exist in Lemma 1 for $\left(p, \widetilde{Z}^{k}, B^{k}\right)$ and let $\Sigma_{t} A^{k t}=\widetilde{Z}_{k}, \Sigma_{k} B^{k}=B$ where $\widetilde{Z}^{k}$ and $Z, B^{k}$ and $B$, satisfy the conditions of Lemma 2 . To execute the matrix $Z$, prescribe trades in period $t$ to be

$$
A^{t}=\Sigma_{k} A^{k t}
$$

By inspection the information contained in (C) is sufficient to allow this to be implemented. By Lemma $1, \widetilde{Z}^{k}=\Sigma_{t} A^{k t}$. By hypothesis and Lemma $2, Z=\Sigma_{k} \widetilde{Z}^{k}$. Therefore, $Z=\Sigma_{t} \Sigma_{k} A^{k t}$ and (E) is satisfied. To establish (A), note that $Z \geqslant-B$ and sign $\tilde{z}_{i n}^{k}=z_{i n}$. From Lemma 1 , it is known that for $\pi^{t}(i)=j$, the trading rule prescribes $a_{i}^{k t}=-a_{j}^{k t}$ and $p \cdot a_{i}^{k t}=0$ for all $t$. Therefore $\Sigma_{k} a_{i}^{k t}=-\Sigma_{k} a_{j}^{k t}[(\mathrm{~A} .2)]$ and $p \cdot \Sigma_{k} a_{i}^{k t}=0[(\mathrm{~A} .3)]$. It only remains for us to show (A.1) to prove the theorem.

By hypothesis, $\widetilde{Z}^{k}$ is a chain, $\operatorname{sign} \tilde{z}_{i n}^{k}=\operatorname{sign} z_{i n}$ and $Z=\Sigma_{k} \widetilde{Z}^{k}$. Choose $B^{k}$ as in Lemma 2. From Lemma 1 , we know that the trading rule applied to ( $p$, $\left.\widetilde{Z}^{k}, B^{k}\right)$ satisfies (A.1) and, therefore, so does this procedure applied to $\left(p, \Sigma_{k} \widetilde{Z}^{k}\right.$, $\left.\Sigma_{k} B^{k}\right)$.

Q.E.D.

Theorem 1 asserts that a rather complicated procedure can be used to achieve full execution of a competitive equilibrium in one round of trade in a barter economy, while fulfilling the conditions of quid pro quo, conservation, and nonnegativity. To summarize the procedure:

(i) The matrix of excess demands is decomposed into a sum of chains. This decomposition is both complex and arbitrary. Without some central direction and notification there is no reason why a trader should have any idea about the chains to which he is assigned and who the other members are.

(ii) Trades are assigned on the basis of (i). At any meeting a pair engages in trade depending on all chains in that period of which both elements of the pair are members. These may be numerous, generating correspondingly complex trades. Assigned trades may include such non-obvious actions as passing up mutually beneficial trading opportunities (when traders meeting are not members of the same chain) and often exchanging for each other goods which neither party to the trade desires. These latter trades are made in anticipation of future trades for desired goods or future trades for goods which, in future trades, will be traded for desired goods, or future trades for goods which in future trades will be traded for goods which in future trades will be traded....

Of course, we have only shown that this complex trading rule is sufficient for (A) and (E) but not that it is necessary. The following section shows that these complications are unavoidable. 


\section{THE IMPOSSIBILITY OF DECENTRALIZING EXCHANGE IN A BARTER ECONOMY}

In this section we shall demonstrate the impossibility of (A), (D), and (E) within a particular class of exchange economies, and this will, of course, suffice to prove Theorem 2. Note that this theorem does not merely say there exists no "plausible" trading rule-e.g., a rule which places limits on the ingenuity or computational capacity of traders or even satisfies some mild continuity condition. It says that within the class of all functions (rules) whose domain is what the traders know and whose range is their sets of feasible trades, there is none which satisfies the stated conditions. It will require that we construct two economies which fulfill the following conditions: (i) under (D) a pair of traders will not be able to determine in which one of the economies they are, and (ii) the necessary conditions for full execution in the two economies are disjoint for the pair of traders (no matter what the other pairs do). ${ }^{12}$

It is desirable to choose the "smallest" example for which (i) and (ii) obtain. Simple calculations show that there will have to be at least three commodities and three traders, and it is not difficult to show that the case of three traders is also too small, no matter what the number of commodities. We shall show that the following trading rule satisfies (A), (D), and (E) for all $(p, Z, B)$ fulfilling (U) if $J \leqslant 3$ :

Trading rule $\gamma$ : For $\pi^{t}(i)=j$, let $a_{i}^{t}=x_{i}^{t}+y_{i}^{t}$ and $a_{j}^{t}=x_{j}^{t}+y_{j}^{t}$ where

$$
x_{i n}^{t}=-x_{j n}^{t}=\left\{\begin{array}{l}
0 \quad \text { if } v_{i n}^{t} v_{j n}^{t} \geqslant 0, \\
\min \left(\left|v_{i n}^{t}\right|,\left|v_{j n}^{t}\right|\right) \quad \text { if } v_{i n}^{t}>0 \quad \text { and } \quad v_{j n}^{t}<0, \\
-\min \left(\left|v_{i n}^{t}\right|,\left|v_{j n}^{t}\right|\right) \quad \text { if } v_{i n}^{t}<0
\end{array} \quad \text { and } \quad v_{j n}^{t}>0,\right.
$$

and $y_{i}^{t}=-y_{j}^{t}$ is constructed according to

$$
\begin{gathered}
{\left[v_{i}^{t}-x_{i}^{t}\right]^{-}<y_{i}^{t}<0 \quad \text { and } \quad p \cdot\left(x_{i}^{t}+y_{i}^{t}\right)=0, \quad \text { if } p \cdot x_{i}^{t}>0,} \\
{\left[v_{j}^{t}-x_{j}^{t}\right]^{-}<y_{j}^{t}<0 \quad \text { and } \quad p \cdot\left(x_{j}^{t}+y_{j}^{t}\right)=0, \quad \text { if } p \cdot x_{j}^{t}>0 .}
\end{gathered}
$$

Part (1) of rule $\gamma$ says, for example, that if $i$ has an excess demand of one unit for a commodity for which $j$ has an excess supply of two units, $j$ is to give one unit to $i$. Trades are made so as to reduce the partners' excess demands to the maximum extent consistent with the dictum "never change the sign of your excess demand."

In the likely event that $p \cdot x_{i}^{t} \neq 0$, say $p \cdot x_{i}^{t}>0$, part (2) says that $i$ may choose from among any commodities for which he still has an excess supply $\left(v_{i n}^{t}-x_{i n}^{t}<0\right)$ and give to $j$ any bundle of those commodities whose value will allow him to maintain quid pro quo. The fact that payment is made in any of the selected set of bundles shows that the rule is compatible with (D) and also accounts for its failure to satisfy (E) when $J \geqslant 4 .{ }^{13}$

${ }^{12}$ See [9, Proposition 5], for an impossibility result similar to Theorem 2.

${ }^{13}$ However, a modification of rule $\gamma$, which introduces a convention as to how to redistribute excess supplies, can be made such that (A), (D), and (E) appear to be compatible for all $(p, Z, B)$ in (U) if $J \leqslant 4$ so that we shall have to admit economies having at least five traders. 
An important consequence of rule $\gamma$ is that if $y_{i n}^{t}>0$, then $j$ may be required to more than fulfill his excess demand for commodity $n$ in order to allow $i$ to maintain quid pro quo-i.e., $j$ must be willing to convert an excess demand into an excess supply. To formalize the opposite, we shall say that a trading rule satisfies $(P)$ at date $t$, if for all $i$ and $n$,

$$
\begin{array}{ll}
v_{i n}^{t} \geqslant v_{i n}^{t+1} \geqslant 0 & \text { if } v_{i n}^{t} \geqslant 0, \\
v_{i n}^{t} \leqslant v_{i n}^{t+1} \leqslant 0 & \text { if } v_{i n}^{t} \leqslant 0 .
\end{array}
$$

Property $(\mathrm{P})$ specifies that for each commodity trade should not change the sign or increase the absolute value of any excess demand. ${ }^{14}$

The relation between $\gamma$ and $(\mathrm{P})$ is as follows:

LeMma 3: For trading rule $\gamma$ to satisfy property $(P)$ at date $t$, it is necessary and sufficient that $a_{i}^{t}=x_{i}^{t}\left(i . e ., y_{i}^{t}=0\right), i=1, \ldots, J$.

Proof: Sufficiency: By inspection if $v_{i n}^{t} \geqslant 0$, then $v_{i n}^{t} \geqslant x_{i n}^{t} \geqslant 0$ and $v_{i n}^{t} \geqslant$ $v_{i n}^{t+1}=v_{i n}^{t}-a_{i n}^{t}=v_{i n}^{t}-x_{i n}^{t} \geqslant 0$. Similarly, if $v_{i n}^{t}<0, v_{i n}^{t} \leqslant x_{i n}^{t} \leqslant 0$ and $v_{i n}^{t} \leqslant$ $v_{i n}^{t+1}=v_{i n}^{t}-x_{i n}^{t} \leqslant 0$.

Necessity: If $a_{i}^{t} \neq x_{i}^{t}$, there is a $y_{i n}^{t} \neq 0$, say $y_{i n}^{t}<0$. From (2) of rule $\gamma, y_{i n}^{t}<0$ implies $p \cdot x_{i}^{t}>0$ and $v_{i n}^{t}<x_{i n}^{t} \leqslant 0$. Since $a_{j n}^{t}=-a_{i n}^{t}=-\left(x_{i n}^{t}+y_{i n}^{t}\right)$, either $v_{j n}^{t} \leqslant 0$, in which case $v_{j n}^{t+1}=v_{j n}^{t}+\left(x_{i n}^{t}+y_{i n}^{t}\right)=v_{i n}^{t}+y_{j n}^{t}<v_{j n}^{t} ;$ or $v_{j n}^{t}>0$, in which case $-x_{i n}^{t}=v_{j n}^{t}$ and $v_{j n}^{t+1}=v_{j n}^{t}+\left(x_{i n}^{t}+y_{i n}^{t}\right)<0$. In both cases, (P) is contradicted.

Q.E.D.

It is to be expected that a demonstration of Theorem 2 will involve economiesunlike those of Theorems 3 and 4-admitting very little slack in their endowments. For a matrix of excess demands $Z$, call the matrix of initial endowments $B$ minimally sufficient for $Z$ if $[Z]^{-}=-B$. If $B$ is minimally sufficient for $Z$, then each trader will want to sell all of his initial endowments in exchange for commodities of which he has none to start.

Rule $\gamma$ will be a basic ingredient in the proof of Theorem 2 because it is the essentially unique method of guaranteeing full execution in economies with three traders whenever endowments are minimally sufficient.

LEMMA 4: For all $(p, Z, B)$ fulfilling $(U)$ such that initial endowments are minimally sufficient $\left(b_{i}=-\left[z_{i}\right]^{-}, i=1, \ldots, J\right)$ and $z \neq 0$ for at most three traders, trading rule $\gamma$ is necessary and sufficient for $(A)$ and $(E)$.

Proof: Necessity: The result is trivial when all $z_{i}=0$ and is readily verified for exactly two $z_{i} \neq 0$, in which case there is a perfect double coincidence of wants. When there are three non-zero vectors $z_{i}$, we can without loss of generality denote them by $i=1,2,3$ and assume that they meet in the sequence $\overline{12}, \overline{13}$, and $\overline{23}$ in periods 1,2 , and 3 , respectively.

${ }^{14}$ In an atemporal context trades satisfying (P) are called excess-demand-diminishing in [11]. Their properties in the present model of a trading economy are studied in [9]. 
For any trading pair $\overline{i j}$, the set of trades consistent with rule $\gamma$ are defined by the restrictions (i) $a_{i}^{t}=-a_{j}^{t}$, (ii) $p \cdot a_{i}^{t}=0$, and for all $n$, (iii) $\left(v_{i n}^{t}-a_{i n}^{t}\right)\left(v_{j n}^{t}-a_{j n}^{t}\right)$ $\geqslant 0$. Condition (iii) says that after $i$ and $j$ perform trades according to $\gamma$, there is no commodity such that $i$ has an unsatisfied excess demand for the commodity and $j$ has an undelivered excess supply. Since (i) and (ii) hold for any admissible trade, $\gamma$ is violated if and only if (iii) is not satisfied. If (iii) is not satisfied by the pair $\overline{12}$ at $t=1$, there must be at least one $n$ such that (a) $v_{1 n}^{2}\left(=v_{1 n}^{1}-a_{1 n}^{1}\right)>0$ and $v_{2 n}^{2}<0$ or (b) $v_{1 n}^{2}<0$ and $v_{2 n}^{2}>0$. Suppose (a); then by the hypothesis that endowments are minimally sufficient and $\Sigma_{i} v_{i n}^{t}=0,\left[v_{1 n}^{2}\right]^{+}=-\left[v_{2 n}^{2}\right]^{-}-\left[v_{3 n}^{2}\right]^{-}>$ $-\left[v_{3 n}^{2}\right]^{-}=-\left[v_{3 n}^{1}\right]^{-}=b_{3 n}$. Since $t=2$ is 1's last opportunity to trade and 3 cannot possibly fulfill all of 1 's excess demand for commodity $n,(\mathrm{~A})$ and (E) are contradicted. Supposing (b), a similar conclusion follows. This shows that $\gamma$ is necessary at $t=1$, and the same arguments may be applied to the trading pair $\overline{13}$ at $t=2$ to show that if $\gamma$ (condition (iii), above) is violated, (A) and (E) will be contradicted. Finally, at $t=3$, (E) implies $v_{2}^{3}=-v_{3}^{3}$ which requires $a_{2}^{3}=v_{2}^{3}-v_{3}^{3}$, exactly what $\gamma$ prescribes.

Sufficiency: This may be constructed directly from the above. However, since $\gamma$ is necessary and by Theorem 1 there always exist trading rules satisfying (A) and $(E)$, it must be sufficient.

Q.E.D.

The informational restrictions imposed by (D.3) will be shown to imply that any pair of traders will not be able to determine whether they are or are not in a three-trader economy. That is, (D.3) requires that they perform the same trade independent of the economy in which they are located. When this is coupled with Lemma 3, the result is a sweeping limitation on the "degrees of freedom" in any successful, decentralized rule. If the rule is to work it must satisfy $\gamma$ and we shall see that except in special cases this will mean that it must violate $(\mathrm{P})$. To prove Theorem 2, it only remains to construct economies where if $(\mathrm{P})$ is violated, full execution is precluded.

Bradley [1] has found an example showing that (A), (D), and (E) are inconsistent for $J \geqslant 5$ if the trading rule satisfies the additional assumption that it is insensitive to a relabelling of commodities-i.e., it is only the amounts of commodities, not their names, which matter. Theorem 4 with its monetary trading rule, below, contradicts Bradley's assumption. The money commodity is definitely in an asymmetric position compared to all other commodities. This could be interpreted as showing the advantages of having at least one commonly recognizable commodity to be used by all traders in settling their accounts. However, suppose, as we have in (D), that all commodities are recognizable. Theorem 2 shows that even when they are, this is not sufficient to guarantee full execution.

\section{An Example that Proves Theorem 2}

Throughout the remainder of this section we shall consider only those economies $(p, Z, B)$ satisfying $(\mathrm{U})$ such that (i) there are five traders and four commodities, (ii) initial endowments are minimally sufficient so that $b_{i}=-\left[z_{i}\right]^{-}, i=1, \ldots, 5$, 
and (iii) equilibrium prices are the same for all commodities, e.g., $p=(1,1,1,1)$. Under conditions (i)-(iii), any economy fulfilling (U) can be completely described by a $5 \times 4$ matrix (of initial excess demands), $Z$, whose row and column sums are zero. Denote by $\mathscr{U}$ the set of all such $Z$.

Also throughout, let the sequence of trading partners in a five-trader economy be $(\overline{12}, \overline{35}, \overline{4}),(\overline{13}, \overline{24}, \overline{5}),(\overline{14}, \overline{25}, \overline{3}),(\overline{1}, \overline{23}, \overline{45})$, and $(\overline{15}, \overline{34}, \overline{2})$ in periods $1-5$ consecutively. This constitutes a round.

Let $\mathscr{L} \subset \mathscr{U}$ be the class of economies representable by matrices of the form

$$
Z=\left[\begin{array}{cccc}
a & 1-a & -b & b-1 \\
0 & 0 & e & -e \\
-a & a-1 & b-e & 1-b+e \\
0 & 0 & 0 & 0 \\
0 & 0 & 0 & 0
\end{array}\right]
$$

where $0 \leqslant a, b \leqslant 1$ and $b-e \geqslant 0$ if $e \geqslant 0$ and $1-b+e \geqslant 0$ if $e \leqslant 0 .{ }^{15}$

Proposition 1: If $Z\left(=V^{1}\right) \in \mathscr{L}$, a necessary condition for $(A)$ and $(E)$ is that $v_{2}^{2}=0$.

Proof: By Lemma 3, $\gamma$ is necessary. By inspection, its application leads to $V_{2}^{2}=0$.

Let $\mathscr{M} \subset \mathscr{U}$ be the class of economies representable by matrices of the form,

$$
Z=\left[\begin{array}{cccc}
a & 1-a & -b & b-1 \\
0 & 0 & e & -e \\
-c & c-1 & d & 1-d \\
c-a & a-c & b-d-e & d+e-b \\
0 & 0 & 0 & 0
\end{array}\right]
$$

where $0 \leqslant a, b, c, d \leqslant 1$ and again $b-e \geqslant 0$ if $e \geqslant 0$ and $1-b+e \geqslant 0$ if $e \leqslant 0$.

The classes $\mathscr{L}$ and $\mathscr{M}$ have been constructed so that by the hypothesis of (D) the pair $\overline{12}$ cannot determine at $t=1$ whether $Z$ belongs to $\mathscr{L}$ or $\mathscr{M}$. The pair must make the same initial trade in each. From Proposition 1 , rule $\gamma$ is necessary if $Z \in \mathscr{L}$. Therefore, if (A), (D), and (E) are to hold for all $Z \in \mathscr{U}$, the pair $\overline{12}$ must adopt it for all $Z \in \mathscr{L} \cup \mathscr{M}$. Since the other traders must be inactive, it follows that $\gamma$ is necessary at $t=1$. The following says that $\gamma$ is necessary for all $t$.

${ }^{15}$ Traders 4 and 5 are "dummies" having no endowments and thus no demands. Their presence in this and the following constructions is a device for minimizing the difficulty in describing the set of admissible trades satisfying (E). When it is recognized that a dummy is a special case of a trader who has already fulfilled his excess demands before trade begins, it will be seen that the overall results would follow a fortiori if we replaced these dummies by "real" traders. 
Proposition 2: A necessary condition for $(A),(D)$, and $(E)$ to hold for all $Z \in \mathscr{U}$ is that if $Z \in \mathscr{M}$, trades must satisfy trading rule $\gamma^{1{ }^{16}}$

Each of the classes $\mathscr{L}$ and $\mathscr{M}$ provided a necessary condition for (A), (D), and (E) to be satisfied for all $Z \in \mathscr{U}$. Next, we introduce another class of economies and with it an additional necessary condition which will be shown to be inconsistent with the above requirements and will, therefore, confirm Theorem 2.

Let $\mathscr{N} \subset \mathscr{U}$ be the class of economies representable by matrices of the following form :

$$
Z=\left[\begin{array}{cccc}
a & 1-a & -b & b-1 \\
1-a & a & b-1 & -b \\
-c & c-1 & d & 1-d \\
c-1 & -c & 1-d & d \\
0 & 0 & 0 & 0
\end{array}\right]
$$

where $0 \leqslant a, b, c, d \leqslant 1$.

Proposition 3: For any $Z \in \mathscr{N}$, a necessary condition for $(A)$ and $(E)$ is that trades satisfy $(P)$ at $t=2$.

So far, it has been established that if $Z \in \mathscr{M}$, trades must satisfy rule $\gamma$ in every period (Proposition 2), and that if $Z \in \mathscr{N}$, trades must satisfy property (P) at $t=2$ (Proposition 3). These two necessary conditions do not, by the next result, coincide.

Proposition 4: There exists no trading rule satisfying $(A)$ such that for all $Z \in \mathscr{M} \cup \mathcal{N}$, the application of trading rule $\gamma$ at $t=2$ will exhibit property $(P)$.

Since a trading rule cannot satisfy (A) and (E) for all $Z \in \mathscr{U}$ while remaining invariant for all $Z \in \mathscr{M} \cup \mathscr{N}$, it only remains for us to show that (D) requires such invariance.

Whether $Z \in \mathscr{M}$ or $\mathscr{N}, z_{1}\left(=v_{1}^{1}\right)$ is defined by the parameters $(a, b)$. The possible changes in trader 1's excess demands and their implications are stated as the following proposition:

Proposition 5 : (i) For all $Z \in \mathscr{M} \cup \mathcal{N}$, if $z_{1}$ is defined by $(a, b)$ and trades satisfy $(A)$, then $v_{1}^{2}$ is of the same form as $v_{1}^{1}\left(=z_{1}\right)$ and is defined by $\left(a, b^{\prime}\right), 0 \leqslant b^{\prime} \leqslant 1$; and (ii) if $(A),(D)$, and $(E)$ are to hold for all $Z \in \mathscr{U}$, the trading pair $\overline{13}$ will not be able to determine at $t=2$ whether $Z \in \mathscr{M}$ or $\mathcal{N}$.

Propositions 1-5 are now combined to prove Theorem 2.

Proof: From Proposition 5, the trading pair $\overline{13}$ will not be able to determine at $t=2$ whether $Z \in \mathscr{M}$ or $\mathscr{N}$. From Proposition 2, if $\mathscr{M}$, trading rule $\gamma$ must be followed; and, from Proposition 3, if $\mathcal{N}$, the trading rule must satisfy (P). From

${ }^{16}$ Propositions 2 through 5 are proved in the Appendix. 
Proposition 4, there will exist economies in both $\mathscr{M}$ and $\mathscr{N}$ in which the application of rule $\gamma$ will fail to satisfy (P). Since (D) requires that the same trading decision must be made in all such cases, whichever of the mutually exclusive alternatives$\gamma$ or $(\mathrm{P})-$ is chosen, it will fail to achieve $(\mathrm{A})$ and $(\mathrm{E})$ in either $\mathscr{M}$ or $\mathcal{N}$. Q.E.D.

\section{MONEY AND COMMODITY INVENTORIES}

The difficulties of barter exchange may be traced to an over-determinacy in the demand for commodities. They are wanted both for final consumption and as a means of payment. Without a double coincidence of wants, these two functions cannot be easily satisfied and each unit of a commodity supplied may have to serve both as a means of payment to one's present trading partner and as an exchange which will satisfy the demands of one's present trading partner's future partners, ..., etc.

Assuming that information beyond that given by (D) is simply not available, some slack in initial endowment is essential to guarantee full execution. When endowments are large, relative to what is minimally sufficient, there are trading rules permitting these two conflicting demands for commodities to be separated in a straightforward, decentralized manner. The very presence of a medium of exchange implies a slackness, that $B$ is not minimally sufficient. Imagine a money economy, with $m$ the money commodity, in a position of equilibrium where traders have no desire to increase or decrease their money balances $\left(z_{i m}=0\right)$. If endowments were minimally sufficient, this would imply $b_{i m}=0$, hardly a description of a money economy. Although the levels of inventories may remain constant on the average, fluctuations from period to period should be regarded as a valuable input in the process of exchange. (Of course, inventories cannot fluctuate if their initial levels are zero.) This is the view taken by R. W. Clower [2], who has emphasized that it is not only inventories of money, but of all commodities, which facilitates exchange. In this section, Theorems 3 and 4 support this contention.

Suppose trader 1's initial endowments are so large that he may fulfill all other traders' excess demands $-b_{1} \geqslant \Sigma_{i \neq 1}\left[z_{i}\right]^{+}$. Consider the following:

Trading rule $\sigma:$ For $\pi^{t}(i)=j$, let

$$
\begin{array}{ll}
a_{i}^{t}=0=-a_{j}^{t} & \text { if } i, j \neq 1, \\
a_{i}^{t}=v_{i}^{t}=-a_{j}^{t} & \text { if } i \neq j=1 .
\end{array}
$$

It is clear that we have the following theorem:

THEOREM 3: If $b_{1} \geqslant \Sigma_{i \neq 1}\left[z_{i}\right]^{+}$, trading rule $\sigma$ satisfies $(A),(D .2)$, and $(E)$.

Trading rule $\sigma$ specifies that trader 1 acts as a clearinghouse. He is the hub of commodity exchange. Other traders look to him and only to him, to purchase their demands and sell their supplies (cf. [11, Lemma 1], though [11] lacks the nonnegativity constraint). 
In order to function as a medium of exchange, money must have a positive value in exchange, a positive price. It has been suggested by some that the major aim in the integration of monetary and value theory is to construct a model in which money has a positive equilibrium price. ${ }^{17}$ This paper represents another tack. For a commodity with positive value, are there any conditions under which it could be usefully employed as a medium of exchange? In this model Theorem 4 answers affirmatively. Suppose there is a commodity, $m$, such that the value of each trader's endowment of it is at least as large as the value of his desired purchases of commodities other than $m-p_{m} b_{i m} \geqslant \Sigma_{n \neq m} p_{n}\left[z_{i n}\right]^{+}, i \in I$. Then consider the following:

Trading rule $\mu$ : For $\pi^{t}(i)=j$, let

$$
a_{i}^{t}=x_{i}^{t}+y_{i}^{t} \quad \text { and } \quad a_{j}^{t}=x_{j}^{t}+y_{j}^{t}
$$

where

$$
x_{i n}^{t}=-x_{j n}^{t}=\left\{\begin{array}{l}
0 \quad \text { if } v_{i n}^{t} v_{j n}^{t} \geqslant 0, \quad \text { or if } n=m, \\
\min \left(\left|v_{i n}^{t}\right|,\left|v_{j n}^{t}\right|\right) \quad \text { if } v_{i n}^{t}>0 \quad \text { and } \quad v_{j n}^{t}<0, \\
-\min \left(\left|v_{i n}^{t}\right|,\left|v_{j n}^{t}\right|\right) \quad \text { if } v_{i n}^{t}<0 \quad \text { and } \quad v_{j n}^{t}>0,
\end{array}\right.
$$

and

$$
y_{i n}^{t}=-y_{j n}^{t}= \begin{cases}0 & \text { if } n \neq m, \\ q \text { where } p \cdot x_{i}^{t}+p_{m} q=0 & \text { if } n=m .\end{cases}
$$

Trading rule $\mu$ assigns a unique, asymmetric role to the commodity $m .{ }^{18}$ The rule says that when traders $i$ and $j$ meet, they should make trades that diminish one's excess supplies and reduce the other's excess demands. Any failure of quid pro quo should be made up in $m$, the commodity which acts as money. Rule $\mu$ should be compared with rule $\gamma$ of Section 4. Parts (1) of each rule are identical except in the treatment of commodity $m$. Part (2) of $\gamma$ says that payment can be made in any assortment of commodities in which the trader has an excess supply, whereas part (2) of $\mu$ narrows the choice of means of payment to commodity $m$ without, however, imposing the restriction that $m$ be in excess supply before it is given up.

THEOREM 4: If $p_{m} b_{i m} \geqslant \Sigma_{n \neq m} p_{n}\left[z_{i n}\right]^{+}, i \in I$, trading rule $\mu$ satisfies $(A),(D .1)$, and $(E)$.

ProOF: The rule requires only a knowledge of the pairs' excess demands and the convention that payment be made in commodity $m$. It satisfies (D.1). (A.3)

\footnotetext{
${ }^{17}$ It seems fairly clear that such a conclusion cannot be obtained from the model of an exchange economy without additional assumptions on the backing or utility of money (Cf. Hahn [4], Kurz [6], Marschak [8], Sontheimer [10], and Starr [12]). The ad hoc nature of these constructs suggest that the problem of integrating monetary and value theory is not equivalent to the demonstration of a positive price for money.

${ }^{18} \mathrm{Cf}$. Clower [3]. Trading rule $\mu$ appears also in [9 and 11].
} 
follows from the definition of trading rule $\mu$, part (2). For goods other than $m$, it will be shown that the rule consisting only of (1), above, satisfies (A.1), (A.2), and $(E)$ and then it will be shown that (2) never contradicts the trades prescribed in (1).

According to (1), if $v_{i n}^{1} \geqslant 0, v_{i n}^{t} \geqslant x_{i n}^{t} \geqslant 0$, and if $v_{i n}^{1}<0, v_{i n}^{t} \leqslant x_{i n}^{t} \leqslant 0$, all $t$. Since $V^{t} \geqslant-W^{t}$, (A.1) is satisfied. (A.2) holds by definition. There is also the implication that if

$$
\begin{aligned}
& v_{i n}^{1} \geqslant 0, \quad \text { then } v_{i n}^{t} \geqslant v_{i n}^{t^{\prime}} \geqslant 0, \quad \text { and if } \\
& v_{i n}^{1}<0, \quad \text { then } v_{i n}^{t} \leqslant v_{i n}^{t^{\prime}} \leqslant 0
\end{aligned}
$$

for $t^{\prime}>t$ and $n \neq m$.

Suppose $v_{i n}^{\tau+1} \neq 0$ for $n \neq m$. Then there is at least one commodity, $n \neq m$, and two traders, $i$ and $j$, such that $v_{i n}^{\tau+1}>0$ and $v_{j n}^{\tau+1}<0$. By hypothesis, there is exactly one period, $s$, when $\pi^{s}(i)=j$. By $(3), v_{i n}^{s} \geqslant v_{i n}^{\tau+1}$ and $v_{j n}^{s} \leqslant v_{j n}^{\tau+1}$. If the traders followed (1), either $\left|v_{j n}^{s}\right| \geqslant\left|v_{i n}^{s}\right|$, in which case $v_{i n}^{s+1}=0$ or $\left|v_{j n}^{s}\right|<\left|v_{i n}^{s}\right|$, in which case $v_{j n}^{s+1}=0$. They cannot both be non-zero, contradicting the assertion that $v_{i n}^{\tau+1} \neq 0$. From (U.1) $p \cdot z_{i}=0$ so $v_{i n}^{\tau+1}=0$, for all $n \neq m$, implies $v_{i m}^{\tau+1}=0$, as well. Thus (E) is fulfilled for all $n$.

By hypothesis

$$
p_{m} b_{i m}=p_{m} w_{i m}^{1} \geqslant p \cdot\left[z_{i}\right]^{+}-p_{m}\left[z_{i m}\right]^{+}=p \cdot\left[v_{i}^{1}\right]^{+}-p_{m}\left[v_{i m}^{1}\right]^{+}
$$

and from (3)

$$
\left[v_{i}^{1}\right]^{+} \geqslant \sum_{t=1}^{t=s}\left[x_{i}^{t}\right]^{+} \quad(s=1, \ldots, \tau)
$$

Therefore

$$
\begin{gathered}
p_{m} w_{i m}^{s}=p_{m} w_{i m}^{1}-p \cdot\left(\sum_{t=1}^{t=s-1} x_{i}^{t}\right) \geqslant p_{m} w_{i m}^{1}-p \cdot\left(\sum_{t=1}^{t=s-1}\left[x_{i}^{t}\right]^{+}\right) \\
\geqslant p_{m} w_{i m}^{1}-p \cdot\left[v_{i}^{1}\right]^{+} \geqslant 0 \quad(s=1, \ldots, \tau+1) .
\end{gathered}
$$

Between the two trading rules, $\sigma$ and $\mu$, and the slack conditions they impose, can we point to one as more efficient than the other? Within the confines of the model, we cannot. Nevertheless, it should be recognized that these qualifying conditions on initial endowments are fundamentally different. One rule requires that the slack be "real" and concentrated in a single agent; the other requires that the slack be in value, concentrated in a single commodity. With trading rule $\sigma$, trader 1 acts as a clearinghouse for excess supplies and demands. But there is a crucial difficulty. Trader 1 cannot perform his function without having, to start, substantial quantities of his own commodities. There is no way of getting around this requirement if trades are to satisfy $(\mathrm{A})$ and $(\mathrm{E}) .{ }^{19}$

\footnotetext{
${ }^{19}$ With only a modest endowment to start, trader 1 could act as the unique redistributor by accepting all commodities offered for sale and filling purchase orders from previously received supplies. Clearly, if quid pro quo is to be maintained in every exchange, full execution will require many more periods than are contained on one round.
} 
In trading rule $\mu$, the amounts $b_{i m}$ are unimportant as long as they are positive and $p_{m}$ is sufficiently large. These features are peculiar to commodity $m$ only. In trading rule $\mu$, the proof of Theorem 4 shows that part (1) of the rule almost suffices to guarantee (A), (D), and (E). It fails only in not satisfying quid pro quo. The sole purpose of trade in commodity $m$ is to establish a counting device to insure that the sum of additions to and subtractions from the value of one's holdings during the course of trade is zero. That the device is embodied in a tangible commodity is clearly inessential. ${ }^{20}$

\section{CONCLUSION}

Jevons writes that difficulties of barter arise because of the absence of double coincidence of wants. ${ }^{21}$

The earliest form of exchange must have consisted in giving what was not wanted directly for that which was wanted. This simple traffic we call barter..., and distinguish it from sale and purchase in which one of the articles exchanged is intended to be held only for a short time until it is parted with in a second act of exchange. The object which thus temporarily intervenes in sale and purchase is money.

The first difficulty of barter is to find two persons whose disposable possessions mutually suit each others wants. There may be many people wanting, and many possessing those things wanted; but to allow an act of barter, there must be a double coincidence which will rarely happen $[5$, p. 3].

Monetary exchange requires only single coincidence: a demander of a commodity encountering a supplier of the commodity and paying the supplier in money. Why should a trader refuse to exchange one excess supply for another? Why is double coincidence regarded as necessary for non-monetary exchange?

In the presence of double coincidence, barter exchange can take place in a fully decentralized way. Given double coincidence, execution of a given redistribution of goods requires traders to consult only their own and their current trading partner's excess supplies and demands and then trade so as to yield up their excess supplies and fulfill their excess demands. In the absence of double coincidence such a trading rule will achieve an inefficient allocation far from competitive equilibrium $[9, \mathbf{1 1}]$. If the trading rule is relaxed to permit sellers to accept payment in commodities for which they have no excess demand, there is always a centralized rule but, in general, no decentralized rule which guarantees full execution in a limited number of trades. Full and decentralized execution is achieved through monetary exchange.

\section{University of California at Los Angeles and}

Yale University

Manuscript received November, 1973.

${ }^{20}$ If it were, it is hard to see how we could have advanced from the abacus to the pencil-and-paper method of doing sums. See [9]. The structure of the present model suggests approaches for further research: introducing specialization in production and exchange, making monetary exchange even more essential; allowing the order of trade to be a matter of choice so that by selling before buying one may choose to reduce the required amount of initial money balances.

${ }^{21}$ Other classical writers including Smith and Mill make virtually the same argument. 


\section{APPENDIX}

Propositions 2 through 5 are restated and proved. Notation and definitions are found in Section 4.

Proposition 2: A necessary condition for (A), (D), and (E) to hold for all $Z \in \mathscr{U}$ is that if $Z \in \mathscr{M}$, trades must satisfy trading rule $\gamma$.

Proof: It is demonstrated in the text that rule $\gamma$ is necessary at $t=1$. Following $\gamma$ at $t=1$ implies $v_{2}^{2}=0$. If at $t=2$ we ignore the presence of trader 2 whose excess demands are zero, we may apply Lemma 4 to obtain the desired result. It only remains to show that if trader 2 does exchange at any date $t>1,(\mathrm{~A})$ and (E) are contradicted. Trader 2 is precluded from exchange at $t=3$ when he meets the dummy trader 5 and at $t=5$, when he meets no one. Clearly, if his first non-zero trade is at $t=4$, (A) and (E) are impossible, since he would then acquire an excess demand which he could not fulfill in the subsequent period of no trade. This leaves $a_{2}^{2} \neq 0$ as the remaining possibility. There appear to be numerous ways of showing a contradiction. One is as follows: assume $e,(a-c)$, and $(d+e-b)$ all greater than zero. Since $v_{2}^{2}=0$ and endowments are minimally sufficient, $w_{2}^{2}=(0,0, e, 0)$. Thus if $a_{2}^{2} \neq 0,2$ must give up some amount, $\delta$, of commodity 3 to 4 , his partner at $t=2$. Since $w_{4}^{2}=w_{4}^{1}=$ $(a-c, 0, d+e-b, 0)$, the only way for 4 to satisfy quid pro quo is to give up $\delta$ of commodity 1 , so $0<\delta \leqslant \min (e, a-c)$.

Now, $v_{11}^{1}=a$ and by non-negativity $v_{11}^{2}=v_{11}^{1}$ and $v_{11}^{3}=v_{11}^{4}=a-c$ and 1's last opportunity to exchange is at $t=3$ when he meets trader 4 . However, because 4 gave up $\delta$ of commodity 1 at $t=2$, $v_{11}^{4}>a-c>w_{41}^{4}=a-c-\delta$. So 1's demand for commodity 1 will remain unfulfilled., Q.E.D.

Proposition 3: For any $Z \in \mathcal{N}$, a necessary condition for $(A)$ and $(E)$ is that trades satisfy $(P)$ at $t=2$.

Proof: $Z \in \mathscr{N}$ is defined by the parameters $(a, b, c, d)$, and since trade at $t=1$ takes place only between traders 1 and 2 redistributing their excess supplies of commodities 3 and $4, V^{2}$ will belong to the class $\mathcal{N}$ with $w_{i}^{2}=-\left[v_{i}^{2}\right]^{-}$and will be defined by the parameters $\left(a, b^{\prime}, c, d\right), 0 \leqslant b^{\prime} \leqslant 1$. For any $V^{2} \in \mathcal{N}$, we shall examine some necessary conditions for $(\mathrm{A})$ and $(\mathrm{E})$.

Let $r_{n}$ be the absolute value of the amount of commodity $n$ exchanged at $t=2$ between $\overline{13}$ and let $s_{n}$ be analogously defined for the pair 24 . Trader 3 cannot give up more of commodity 1 than $w_{31}^{2}=w_{31}^{1}$ $=c$. If $c>a$ and $c \geqslant r_{1}>a$, trader 3 will have on hand at the start of the fourth period only $c-r_{1}$ of commodity 1 to meet trader 2's excess demand which, at the very least, is $1-a-(1-c)=c-a$. Since $c \geqslant r_{1}>a$, and $t=4$ is 2's last trading opportunity, (A) and (E) are contradicted. Therefore, $r_{1} \leqslant \min (a, c)$ which implies that trader 1 should not take more of commodity 1 than will fulfill his excess demand. Reasoning along the same lines yields $s_{2} \leqslant \min (a, c) ; r_{2}, s_{1} \leqslant \min (1-a, 1-c)$; $r_{3}, s_{4} \leqslant \min \left(b^{\prime}, d\right) ;$ and $r_{4}, s_{3} \leqslant \min \left(1-b^{\prime}, 1-d\right)$.

The above inequalities on $r_{n}$ and $s_{n}$ prohibit a positive excess demand from being increased or converted into a negative. Also shown above was that if $Z \in \mathcal{N}$, endowments at $t=2$ would be minimally sufficient for excess demands which makes it impossible by (A) for a negative excess demand to be increased (in absolute value) or converted into a positive. These conditions define (P). Q.E.D.

Proposition 4: There exists no trading rule satisfying $(A)$ such that for all $Z \in \mathscr{M} \cup \mathscr{N}$, the application of trading rule $\gamma$ at $t=2$ will exhibit property $(P)$.

Proof: For all $Z \in \mathscr{M} \cup \mathcal{N}$, if trades at $t=1$ satisfy (A), then $v_{1}^{2}$ must be of the form $\left(a, 1-a,-b^{\prime}\right.$, $\left.b^{\prime}-1\right), 0 \leqslant b^{\prime} \leqslant 1$, and $v_{3}^{2}=v_{3}^{1}$ must be $(-c, c-1, d, 1-d)$. Since $\overline{13}$ are trading partners at $t=2$, apply the results of Lemma 3 to exhibit the necessary and sufficient conditions under which rule $\gamma$ and property $(\mathrm{P})$ will overlap. They are as follows:

$$
\min (a, c)+\min (1-a, 1-c)=\min \left(b^{\prime}, d\right)+\min \left(1-b^{\prime}, 1-d\right)
$$

which describes that coincidence where excess demands can be reduced to the maximum extent (satisfying part (1) of $\gamma$ ) without additional trade to maintain quid pro quo (not violating (P)). Such an exact state cannot always obtain.

Suppose the contrary. Then, it must be true that for all $0 \leqslant a, b, c, d \leqslant 1$, there exists $b^{\prime}, 0 \leqslant b^{\prime} \leqslant 1$, satisfying $\left(^{*}\right)$ above. But, for all $b^{\prime}, 0 \leqslant b^{\prime} \leqslant 1$,

$$
\min \left(b^{\prime}, d\right)+\min \left(1-b^{\prime}, 1-d\right) \geqslant \min (d, 1-d) ;
$$


and, for any $\delta, 0 \leqslant \delta \leqslant 1$, there exist values of $a$ and $c, 0 \leqslant a, c \leqslant 1$, such that

$$
\delta \geqslant \min (a, c)+\min (1-a, 1-c) .
$$

Since $a, c$, and $d$ are independent, they may be chosen such that $\min (d, 1-d)>\delta$, and condition $\left(^{*}\right)$ is not satisfied.

Q.E.D.

Proposition 5: (i) For all $Z \in \mathscr{M} \cup \mathcal{N}$, if $z_{1}$ is defined by $(a, b)$ and trades satisfy $(A)$, then $v_{1}^{2}$ is defined by $\left(a, b^{\prime}\right), 0 \leqslant b^{\prime} \leqslant 1$; and (ii) if $(A),(D)$, and $(E)$ are to hold for all $Z \in \mathscr{U}$, the trading pair $\overline{13}$ will not be able to determine at $t=2$ whether $Z \in \mathscr{M}$ or $\mathscr{N}$.

Proof: (i) For trader 1 , whether $\mathscr{M}$ or $\mathcal{N}$, at $t=1$ he can only trade in commodities 3 and 4 . If $\mathscr{M}$, then $a_{1}^{1}=(0,0, x,-x)$ where $\min (e, 0) \leqslant x \leqslant \max (e, 0)$. Since $v_{1}^{2}=v_{1}^{1}-a_{1}^{1}$, set $b^{\prime}=b-x$ and obtain $0 \leqslant b^{\prime} \leqslant 1$. If $\mathscr{N}$, then $a_{1}^{1}=(0,0, x,-x)$ where $-b \leqslant x \leqslant 1-b$. Again, set $b^{\prime}=b-x$ to get $0 \leqslant b^{\prime} \leqslant 1$.

(ii) From Proposition 2, it has been shown that if $Z \in \mathscr{M}$, trader 1 must set $b^{\prime}=b-e$. Therefore, if $Z \in \mathscr{N}$, and trader 1 changes from $b$ to any $\bar{b}, 0 \leqslant b \leqslant 1$, there exists $Z \in \mathscr{M}$ with $e$ such that $\bar{b}=b-e$. For trader $3, a_{3}^{1}=0$ for all $Z \in \mathscr{M} \cup \mathcal{N}$. Since traders can only recall their previous trades and not their previous partners' excess demands, the observations made by 1 and 3 in $\mathscr{M}$ or $\mathscr{N}$ are indistinguishable at $t=2$.

Q.E.D.

In the proofs of Propositions 1-5, care has been taken to show that the results hold when the parameters satisfy inequality rather than equality conditions. This suggests that Theorem 2 holds for a positive fraction of all $Z \in \mathscr{U}$. It would be interesting to inquire whether the fraction of economies for which there exists no decentralized rule leading to full execution increases with the number of individuals and commodities.

\section{REFERENCES}

[1] Bradley, Gordon: "Trading Rules for a Decentralized Exchange Economy," in Symposium on the Theory of Scheduling and its Applications, ed. S. E. Elmaghrabig. New York: SpringerVerlag, 1973.

$\rightarrow$ Clower, Robert W.: "Is There an Optimal Money Supply?" The Journal of Finance, 25 (1970), 425-433.

[3] — : "A Reconsideration of the Microfoundations of Monetary Theory," Western Economic Journal, 5 (1967), 1-8.

[4] Hahn, Frank: "On Some Problems of Proving the Existence of Equilibrium in a Money Economy," in The Theory of Interest Rates, ed. F. H. Hahn and F. P. R. Brechling. London: Macmillan, 1965.

[5] Jevons, William S.: Money and the Mechanism of Exchange. London: D. Appleton and Co., 1875

[6] Kurz, MordecaI: "Equilibrium with Transactions Cost and Money in a Single-Market Exchange Economy," Technical Report No. 51, Institute for Mathematical Studies in the Social Sciences, Stanford University, January, 1972.

[7] Maclane, Saunders, and Garrett Birkhoff: Algebra. New York: Macmillan, 1967.

[8] MarschaK, JACOB: "The Rationale of Money Demand and of 'Money Illusion'," Metroeconomica, 2 (1950), 71-100.

[9] Ostroy, JosePh M.: “The Informational Efficiency of Monetary Exchange," American Economic Review, 63 (1973), 597-610.

[10] Sontheimer, Kevin: "On the Determination of Money Prices," Journal of Money, Credit, and Banking, 4 (1972), 489-508.

[11] Starr, Ross M.: "The Structure of Exchange in Barter and Monetary Economies," Quarterly Journal of Economics, 88 (1972), 290-302.

[12] — : "The Price of Money in a Pure Exchange Monetary Economy with Taxation," Econometrica, 42 (1974), 45-54. 